\title{
Nagel-Schreckenberg Model of Traffic - Study of Diversity of Car Rules
}

\author{
Danuta Makowiec and Wiesław Miklaszewski \\ Institute of Theoretical Physics and Astrophysics, Gdańsk University, \\ ul. Wita Stwosza 57, 80-952 Gdańsk, Poland
}

\begin{abstract}
The Nagel-Schreckenberg model of traffic is modified by the assumption that each car has an individual velocity limit. By simulations, the effect of supplementary rules is checked: (a) a speed limit of the slowest car is changed and/or (b) a speed limit of a car with zero gap behind is increased. It is shown that both rules increase the mean velocity; (b) rule influences the character of congested traffic - cars move though at low velocity.
\end{abstract}

\section{Introduction}

Recently a number of cellular automata models have been proposed in order to investigate the dynamical aspects of the traffic system [1,2]. There are two basic cellular automata models describing single lane traffic flow: the NagelSchreckenberg (NaSch) model [3] and the Fukui-Ishibashi one [4. The cellular automata traffic models, although they suffer from some limitations (see [5] for details), reproduce important real life traffic phenomena such as spontaneous formation of jams [6], explain the impact of global traffic light control strategies [7. and conditions for car accidents [8,9]. In the framework of NaSch model, several very specific real life observations have been attempted to simulate. Let us mention the German highway rules [10, different rules for overtaking [1] or even an individual driver reaction to traffic [11].

The road network is rather poor in Poland - the network is sparse and roads are often in a bad condition. Plenty of different vehicles: bikes, horse carts, farming or construction machines and all kinds of modern cars meet together on the same road. Since usually there is no chance for overtaking, then a driver going behind slowly moving vehicles, is getting nervous and often forces the vehicle ahead to change its driving. Then usually such a hindrance vehicle (a) pulls over to let another vehicle to pass or (b) speeds-up. By this paper we implement (b) behavior by advanced NaSch model. To model car diversity we propose to violate of the common speed limit. However, when we only relax the speed limit then, obviously, a stationary state is determined by the slowest vehicles. There is a need to supplement the model by rules which influence the slowly moving cars. The extra rules proposed by us are again related to Polish drivers common habits. The first rule effects in hanging the speed limit of the slowest car. The second rule increases the speed limit of each car which distance 
to a following one is 0 . Here the velocity adjustment is based on the distance to a car that follows - a looking behind rule [12].

In the next Section we repeat the definition of the basic model, since details of NaSch model may influence results 13 . Section 3 describes the simulation procedure. We report our results concerning time development and properties of stationary states in Section 4. The final section contains summary and propositions for further model development.

\section{NaSch Model}

The probabilistic cellular automata model of traffic represents a lane as onedimensional lattice of $L$ cells. Each cell is either occupied by one of $N$ cars or is empty. A car can move with the velocity determined by integer values bounded by a speed limit $v_{\max }$. At time $t$ a car is identified by a cell number $x_{i}^{(t)}$ occupied by the car and its velocity $v_{i}^{(t)}$. The number of empty cells before the car is called a gap and denoted $g_{i}^{(t)}=x_{i+1}^{(t)}-x_{i}^{(t)}-1$. The cars move along the lane according to rules related to the driving habits:

- acceleration: $v_{i}^{(t+1)}=\min \left(v_{i}^{(t)}+1, v_{\max }\right)$;

- deceleration: $v_{i}^{(t+1)}=\min \left(v_{i}^{(t)}, g_{i}^{(t)}\right)$;

- randomization: if random $<p$ then $v_{i}^{(t+1)}=\max \left(v_{i}^{(t)}-1,0\right)$;

- movement: $x_{i}^{(t+1)}=x_{i}^{(t)}+v_{i}^{(t+1)}$.

At each discrete time step $t \rightarrow t+1$ the positions and velocities of all cars are synchronously updated.

The relation to empirical results is established by the notion of mean traffic flow $J=\langle v \rho\rangle$ for a given car density. In case of NaSch model, this flow depends on car density and deceleration probability $J=J(\rho, p)$. Relation between vehicle density $\rho$ and flow $J$ is called the fundamental diagram. At low density, $\rho \ll 1$, the flow is characterized by a linear dependence on vehicle density - a free flow state. At high densities, the flow decreases with increasing density. Vehicles are said to be in a congested state - stop-and-go waves dominate in dynamics of the system.

\section{Simulation Procedure}

Our simulations are performed on one-dimensional lattice of length $L=10000$ sites with periodic boundary conditions. Such a system size is large enough to reduce finite-size effects [5]. If $N$ denotes a number of cars then car density $\rho$ is defined as $\rho=N / L$. Each simulation starts at random initial conditions: random localization of cars on a lane and random initial velocities. For each initial configuration, we update the individual vehicle velocity and position in accordance with the update rules described in the previous section. By observing first $L$ time steps we obtain a picture of the development in time of a given system property while averaging over next $L$ time steps we get the mean value of the 
property in a stationary state. Each simulation experiment is repeated for a number (100) of different realizations.

\section{Individual Velocity Limit}

\subsection{Definitions of Supplementary Rules}

Let us assign an individual speed limit to each driver: $v_{\max }(i), i=1, \ldots, N$ and $v_{\text {max }}(i) \in 1,2, \ldots, v_{\text {lim }}$. Let us denote this model as $(\mathbf{0}, \mathbf{0})$. The car flow $J(\rho, p)$ is determined by slowly moving vehicles due to the hindrance effect of other cars. It means that in a stationary state, all cars are moving in a few clusters which are led by cars with the speed limit equal to 1 . Therefore we modify the model: at each time step one car chosen from of all cars driving with the smallest actual velocity, obtains a new maximal velocity at random. Let us denote this rule as $(\mathbf{1}, \mathbf{0})$.

In Fig. 1 the resulting flow is shown for all values of $\rho$ and $p$ simulated by us. The fundamental diagrams having the usual form, for two particular values of the randomization parameter $p$ (important for further simulations), are presented in Fig. 2. The linear dependence of traffic flow on car density is observed in one third of the density interval. But the mean velocity of the traffic is reduced considerable, as if the basic NaSch model with $v_{\max }=1$ was simulated.

The following modifications are proposed to accelerate traffic:

$(\mathbf{2}, \mathbf{0})$ : a new velocity limit, assigned to the slowest car, must be greater than the car previous limit

$(\mathbf{0}, \mathbf{1})$ : if a gap between two subsequent cars is equal to 0 , then the speed limit of the car ahead is increased by 1 . The new speed limit cannot excess the overall limit $v_{l i m}$.

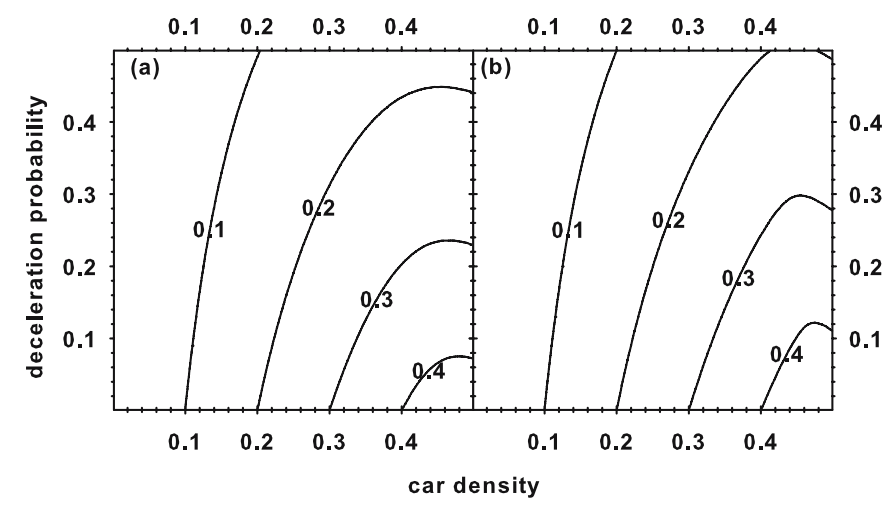

Fig. 1. Traffic flow when each vehicle has its own speed limit $v_{\max }(i)$ chosen at random from $1, \ldots, 10$ (left panel) and from $1, \ldots, 90$ (right panel) where the slowest driving vehicle changes the speed limit to a new one - model $(1,0)$. 


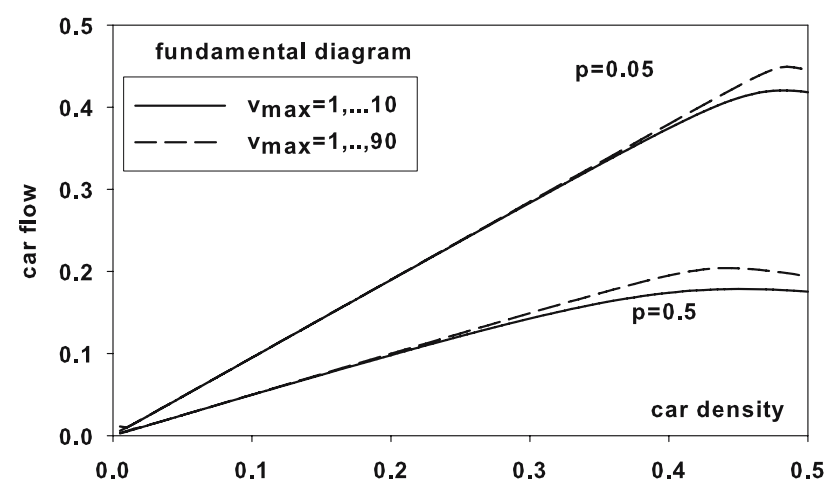

Fig. 2. The fundamental diagram for $(1,0)$ model in case $v_{\text {lim }}=10$ and $v_{\text {lim }}=90$ for $p=0.05$ and $p=0.5$. Note that in case of the NaSch model with $v_{\max }=1, \rho=0.5$, resulting flow corresponds to maximal flow.

The combination of the above notations means that the corresponding combination of the rules is applied simultaneously. For example, $(1,1)$ means that the slowest driving car changes the speed limit and all vehicles, which distance to a car behind is zero, have their speed limit increased by 1 .

\subsection{Results}

Starting from random initial states with individual velocity limits taken at random from the interval $1,2, \ldots, v_{l i m}$, we observe the development of mean velocity and mean maximal velocity. The model parameters are chosen in a way which allows us to investigate different aspects of road conditions:

(A) - basic traffic: $\rho=0.01 p=0.05$ and $v_{\text {lim }}=10$,

(B) - high car density: $\rho=0.1 p=0.05$ and $v_{\text {lim }}=10$,

(C) - high car diversity: $\rho=0.01 p=0.05$ and $v_{\text {lim }}=90$,

(D) - high randomization: $\rho=0.01 p=0.5$ and $v_{\text {lim }}=10$.

The time evolution of the mean velocity and changes of the average maximal velocity are shown in Fig. 3. The time axis is logarithmic to better visualize different features of subsequent time intervals.

When the car density is low, the supplementary rules accelerate the traffic in short times what, after many time steps, lead to mean velocities higher than $(0,0)$ rule provides. However, this occurs to all rules except one: $(1,0)$ rule. After many time steps only this rule leads to the mean speed limit smaller than in $(0,0)$ model. In general, it appears that the mean maximal velocity increases for all rules except $(1, *)$ rules. This is caused by our update configuration algorithm. The most left Of slowest cars on a lane is chosen to alter its sleep limit. After many time steps, cars drive with the same speed in jammed clusters. Since the most left car is often a car from a tail of a cluster, it should have a high speed limit. Therefore, a random change means here the exchange of a high speed limit to some other one, probably 

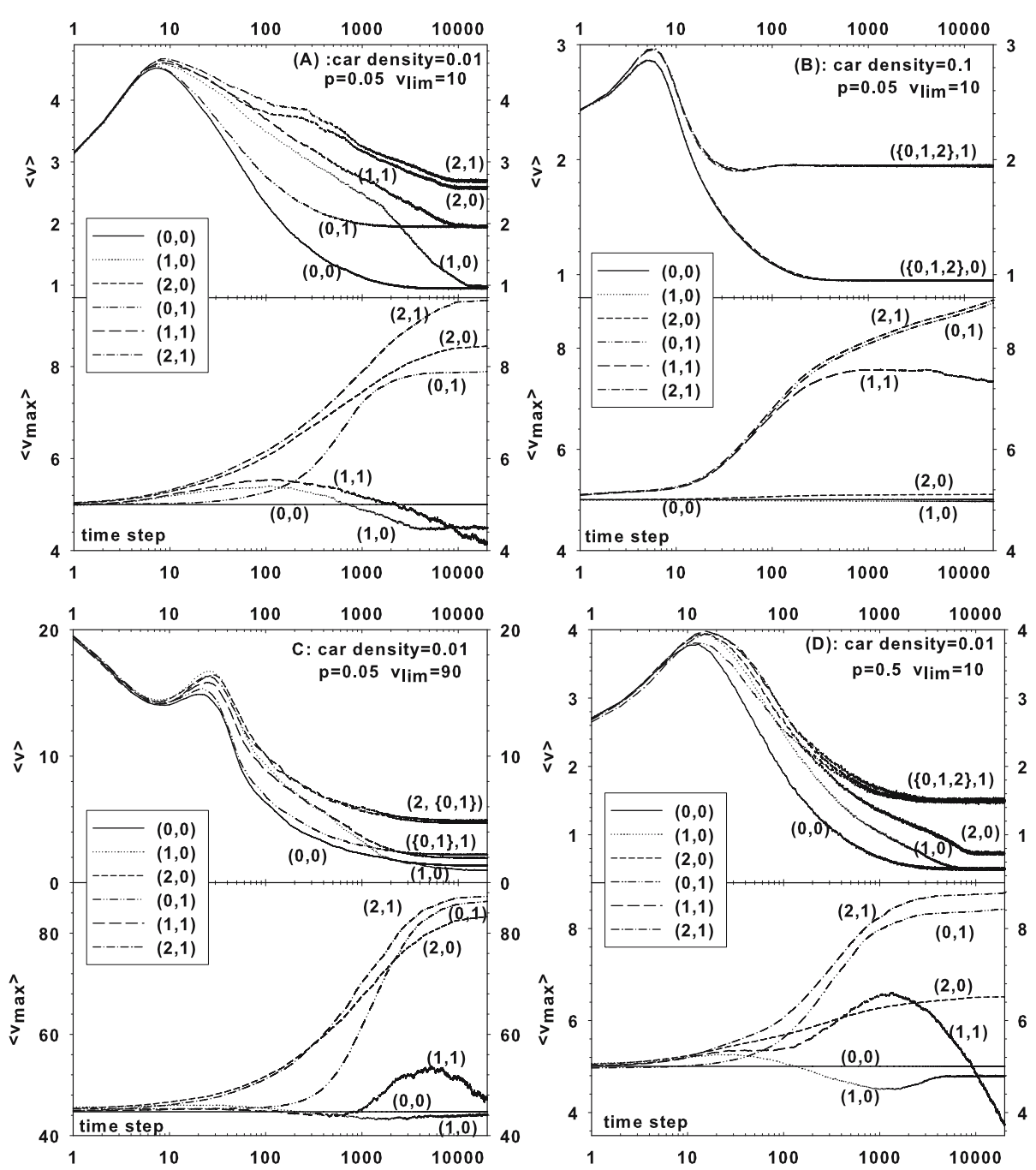

Fig. 3. Time development of the mean velocity $\left\langle v^{(t)}\right\rangle$ and mean maximal velocity $\left.\left\langle v_{\max }^{(t)}\right\rangle\right)$ when each vehicle has its own maximal velocity $v_{\max }(i)$. The label at a curve indicates the rule applied.

lower. After long evolution, the decrease of the mean speed limit is observable. Our simulations show that in the case (D) $\left\langle v_{\max }\right\rangle$ does not stabilize within $10^{5}$ time steps though the mean velocity stabilizes after $10^{4}$ time steps.

The rules: $(*, 1)$, independently on the car density, lead to the mean traffic velocity higher than $(0,0)$ model. When a car density is small then also $(2,0)$ rule is efficient in establishing faster traffic. 

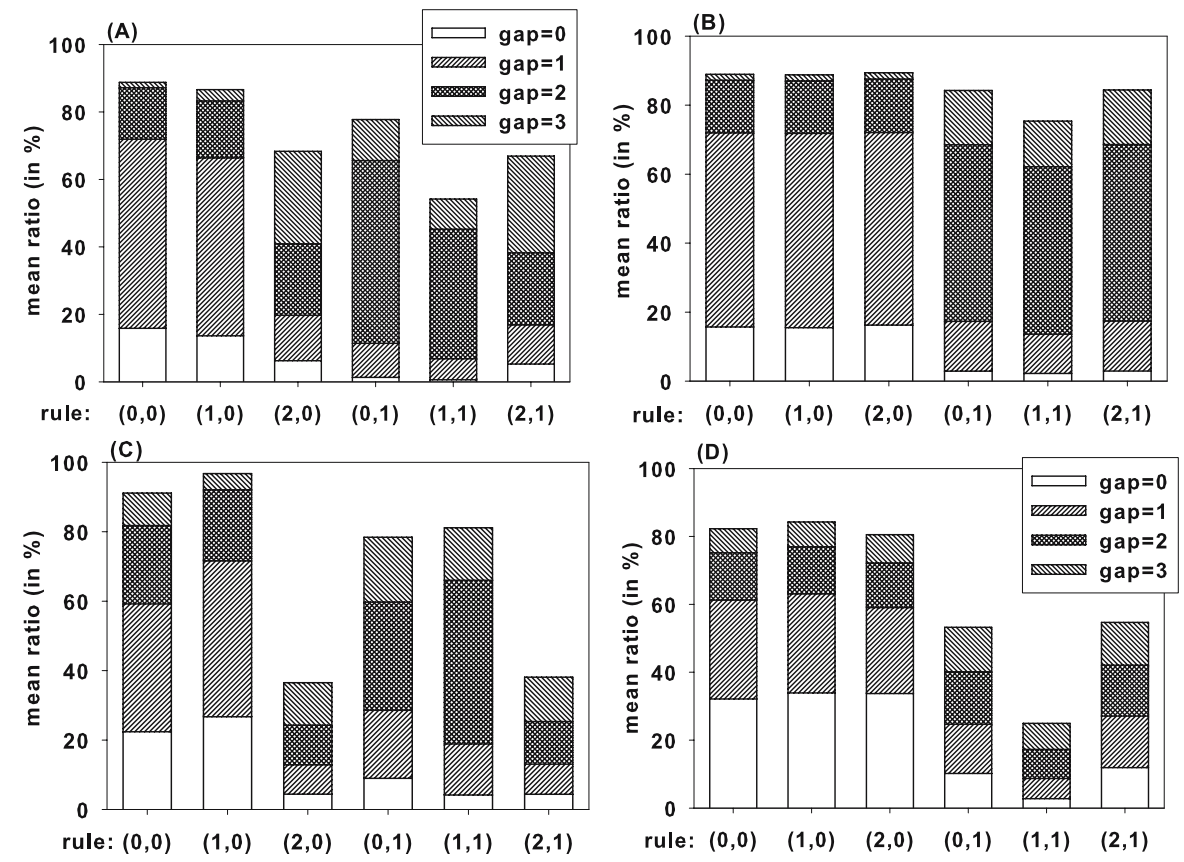

Fig. 4. Distribution of small gaps for different road conditions (A) - (D) and different rules

In all road cases: $(\mathrm{A})-(\mathrm{D})$ and for $(2,1)$ and $(0,1)$ rules, the mean speed limits significantly increase to values close to $v_{\text {lim }}$. However, this high mean speed limits only little influence mean velocities. (This property is present also in the model with $(2,0)$ rule at low car density). Thus, although drives are allowed to go fast, they drive at about one fourth of a speed limit. To find explanation of this discrepancy we examine the structure of a stationary configuration. In particular, we concentrate one a distribution of small gaps: $g_{i}=0,1,2,3$. The series of plots in Figs. 4 present mean percentages of small gaps in stationary configurations.

Gaps 0 and 1 are crucial in establishing the stop-and-go state of congested traffic. The two rules: $(0,0)$ and $(1,0)$, independently on road conditions, lead to a state where more than $60 \%$ of vehicles are trapped in the stop-and-go phase; only few cars are separated more than 3 empty cells.

The rules $(*, 1)$ stabilize on configurations where stopped cars occur rarely. It meanss that the $(0,1)$ part of this rules is applied rarely. Thus there are no 2 -cell clusters on a lane filled by two cars. Note that in all road condition the system stabilizes on configurations in which more than $90 \%$ cars are separated by empty cells. It means that even in case (B) of high car density, almost all vehicles are moving.

In case of high randomization (D) only about $25 \%$ of vehicles are separated by small gaps. It appears that the distribution of large gaps exibits a powerlaw dependence on a gap size, see Fig. 5(a). Each time one meets a power-law 

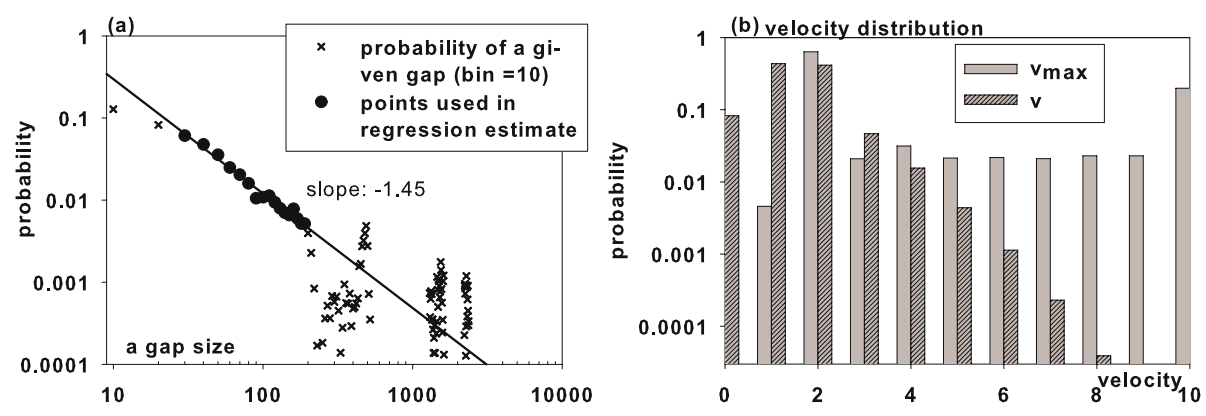

Fig. 5. (a) Log-log plot of distribution of large gaps in case of a model with $(1,1)$ rule and (D) parameters. (b) Histograms of actual car velocity and speed limits for a model with $(1,1)$ rule and (D) parameters. Subsequent bars correspond to subsequent 2000-time-step counting started after 10000 initial time steps. (log scale)

dependence, a suggestion is born that a stable configuration is critical in some sense [14. In Fig. 5 (b) a distribution of actual car velocities is confronted with a distribution of speed limits. Note that the speed limit for more than $80 \%$ of cars is 2 . Hence the slow mean traffic velocity is related to the speed limit distribution. The criticality emerges from a delicate balance between effects of the parts of $(1,1)$ rule which act oppositely to the speed limit: after many time steps, $(1,0)$-part works systematically on decreasing $\left\langle v_{\max }\right\rangle$ while $(0,1)$-part at high $p$ is effective in increasing $\left\langle v_{\max }\right\rangle$.

\section{Summary}

In the presented paper we investigated effectiveness of rules mimicing habits popular among drivers on Polish roads. Computer experiments were performed on the base of NaSch traffic model. The car diversity was achieved by violation of the car speed limit. The first specific supplementary rules $(*, 0)$ were examples of global rules - the speed limit of a single slowest car was modified, while the second ones $(*, 1)$ were a kind of local rules - the speed limit is changed to each car which stopped another car.

Simulations exemplify the basic expected result - slowly moving vehicles determined traffic. While effect of $(*, 0)$ rules on the mean traffic velocity was small but the insistence of the car behind in speeding-up of a slow vehicle (rules: $(*, 1)$ ), increased the mean velocity. We also found that the character of congested traffic was different in case of $(*, 1)$ rules - the stop-and-go waves were absent. Cars were driving permanently though at low velocity.

Long runs magnified some details of our algorithms. For example, in case of $(1,1)$ rule we could observe emerging of properties which are characteristic for dynamical systems being in a critical state.

In future simulations, the algorithm of changing a speed limit of one of the slowest cars, should be modify in a way to avoid decrease of maximal velocity, 
e.g., a car located as most right on a lane should alter its limit. On the other hand, this slowest car could be shifted to the end of a cluster behind to imitate pulling over of a hindrance to let other vehicles to pass.

\section{Acknowledgments}

We acknowledge the support of Gdańsk University - project BW-5400-5-0166-5 and Academic Computer Centre in Gdansk for CPU access.

\section{References}

1. Chowdhury, D., Santen, L., Schadschneider, A.: Statistical physics of vehicular traffic and some related systems. Phys. Rep. 329 (2000) 199-329

2. Helbing, D.: Traffic and related self-driven many-particle systems. Rev. Mod. Phys. 73 (2001) 1067-1141

3. Nagel, K., Schreckenberg, M.: A cellular automaton model for freeway traffic. J. Phys. I France 2 (1992) 2221-2229

4. Fukui, M., Ishibashi, Y.: Traffic flow in 1d cellular automaton model including cars moving with high speed. J. Phys. Soc. Jpn. 65 (1996) 1868-1870

5. W. Knospe, L. Santen, A.S., Schreckenberg, M.: An empirical test for cellular automaton models of traffic flow. Phys. Rev. E 70 (2004) 016115-1-016115-25

6. Knospe, W., Santen, L., Schadschneider, A., Schreckenberg, M.: Towards a realistic microscopic description of highway traffic. J. Phys. A 33 (2000) L477-L485

7. Brockfeld, E., Barlovic, R., Schadschneider, A., Schreckenberg, M.: Optimizing traffic lights in a cellular automaton model for city traffic. Phys. Rev. E 64 (2001) 056132

8. Boccara, N., Fukś, H., Zeng, Q.: Car accidents and number of stopped cars due to road blockage on a one-lane highway. J. Phys. A 30 (1997) 3329-3332

9. Moussa, N.: Car accidents in cellular automata models for one-lane traffic flow. Phys. Rev. E 68 (2003) 036127

10. Ebersbach, A., Schneider, J., Morgenstern, I.: Simulation traffic on german highways based on the nagel-schekenberg-model. Int. J. Mod. Phys. C 12 (2001) 10811089

11. Moussa, N.: Cellular automata for traffic flow with "slow-to-start" rule: Effect of randomization. Int. J. Mod. Phys. C 15 (2004) 29-43

12. Nakayama, A., Sugiyama, Y., Hasebe, K.: Effect of looking at the car that follows in an optimal velocity model of traffic flow. Phys. Rev. E 65 (2001) 016112-106112-6

13. Xue,Y., Dong,L., Li, L., Dai, S.: Effects of changing ordres in the update rules on traffic flow. Phys. Rev. E 71 (2005) 026123-1-026123-6

14. Bak,P., Tang,C., Wiesenfeld, K.: Self-organized criticality. Phys. Rev. A 38 (1988) 365-374 